\title{
The homotopy perturbation solution for the Boltzmann equation of solid-gas flow with one-dimensional BGK model
}

\author{
Chang-jiang Liu ${ }^{a}$, Shao-peng Yang ${ }^{\text {}}$, Qiang Xu,and Yun-jie Qing \\ State Key Laboratory of Geohazard Prevention and Geoenvironment Protection, Chengdu \\ University of Technology, Chengdu 610059, China \\ a changjiangliucd@126.com, byangshaopeng01@yeah.net
}

Keywords: Two-phase Flows, Boltzmann Equation, Homotopy Perturbation, BGK Model.

Abstract. The motion of many solid-gas two-phase flows is often characterized by the Boltzmann equation. In order to simplify the Boltzmann equation, the one-dimensional Bharnagar-Gross-Krook (BGK) model is applied to replace the collision term while reserving the convective-diffusion term. Then, the homotopy perturbation method (HPM) is utilized to solve the simplified Boltzmann equation to obtain the approximate analytical solution. The results show that the analytical solution satisfies all the constraint conditions, and its structure is in accord with the structure of the solution that is obtained by traditional Chapman-Enskog method, and moreover, the solution process of HPM is much more simple and convenient.

\section{Introduction}

The scientific development of two-phase flow theory begins in the 1950s. There are two theories for studying the two-phase flow; one is the traditional macroscopic continuum theory, the other is the rapid developing microcosmic kinetics theory [1]. Then the microcosmic kinetics theory can be classified into two types, one is the kinetics method based on the Boltzmann equation, the other is the method of probability density function (PDF) transport equation based on single particle motion equation $[2,3]$.

Macroscopic continuum theory is the principal theory for the study of two-phase flow, and it requires any micro unit containing numberless particles [4]. Generally, if the molecule size of the fluid phase is very small, we can apply continuum theory to study it, but for the larger solid particle, it is unable to apply continuum theory to describe the inter-collision of particles [5]. Therefore, the solid particle of solid-gas two-phase flow can be regarded as the molecule in gas kinetic theory, and its motion can be described by the Boltzmann equation. For instance, the dry sturzstrom of high-speed distant landslide that is generally caused by strong earthquake can be regarded as a solid-gas two-phase flow, and the motion of its solid sturzstrom particles can be described by the Boltzmann equation. The Boltzmann equation is as follows:

$$
\frac{\partial f}{\partial t}+v_{i} \cdot \frac{\partial f}{\partial x_{i}}+\frac{\partial X_{i} \cdot f}{\partial v_{i}}=\left(\frac{\partial f}{\partial t}\right)_{c o l l}
$$

where $f=f\left(x_{i}, v_{i}, t\right)$ denotes the velocity distribution function of single particle; $x_{i}$ denotes the space coordinate; $v_{i}$ denotes the velocity of particle; $t$ denotes the time coordinate; $X_{i}$ denotes the external force acting on the particles of unit mass, and it depends on $v_{i}$; if only the gravity act on the particle, $X_{i}$ is namely gravitational acceleration $\mathrm{g}_{i}$, and doesn't depend on $v_{i} ;\left(\frac{\partial f}{\partial t}\right)_{\text {coll }}$ denotes the change rate of distribution function cased by inter-collision of particles.

Because the Boltzmann equation is a complex differential and integral nonlinear equation, it is very hard to solve it, and it is also a frontier subject in recent years. The main methods for solving the Boltzmann equation are variation method, Chapm-Enskog perturbation method and Grad-13 moments 
method. Recently, the researches of the Boltzmann equation become diversification and perfection. Huang and Ding [6] solved the Boltzmann equation with small Knudsen parameter by singular perturbation method, and the regular solution, initial layer solution and boundary layer solution were obtained. Zhang et al. [7] applied homotopy analysis method to solve the Boltzmann equation of dilute solid-liquid two-phase flows and the first-order approximate solution was obtained. These researches advanced the proceeding for solving the Boltzmann equation. Moreover, Monte Carlo method, test particle method, molecule kinetics method, direct modeling Monte Carlo method, energy method and so on dramatically advanced the numerical modeling solving of the Boltzmann equation [8]. But all of these methods need some additional optimization conditions, and its derivation process is very complex. The simple and clear analytic solution of original Boltzmann equation has not been obtained.

The homotopy perturbation method (HPM) introduced by He [9] is an effective method to solve strong nonlinear problems. It is widely applied to mathematics, mechanics, economics, and biology and so on [10-12]. This method does not depend on the small parameter. We can obtain the solution with enough precision regardless of the nonlinear equation containing a small parameter. Therefore, we solved the Boltzmann Equation with one-dimensional BGK models and the first-order approximate analytic solution was obtained in this paper.

\section{The HPM solution for the Boltzmann equation with BGK model}

Equation (1) was widely applied to describe the rapid granular flow, solid-gas two-phase flow and solid-liquid two-phase flow. Wang and $\mathrm{Ni}$ [1] neglected the collision term and time-dependent term to simplify the Boltzmann equation. We reserve the convective-diffusion term and replace the collision term by the BGK model in the Boltzmann equation to simplify it, and the simplified Boltzmann equation is

$$
\frac{\partial f}{\partial t}+v \cdot \frac{\partial f}{\partial x}+X \frac{\partial f}{\partial v}=-\hat{v}\left(f-\hat{f}^{(0)}\right)
$$

where $\hat{f}^{(0)}=\frac{C}{(2 \pi T)^{1 / 2}} \exp \left(-\frac{v^{\prime 2}}{2 T}\right)$ denotes local Maxwell velocity distribution function; $\hat{v}$ denotes collision frequency; $v^{\prime}=v-\langle v\rangle$ denotes peculiar velocity; $v$ denotes the velocity of particle; $\langle v\rangle$ denotes the average velocity of particle; $C$ denotes the volume specific concentration of particle; $T$ denotes the temperature of particle. $C,\langle v\rangle$ and $T$ depend on $x$ and $t$.

Assume that Eq. (2) is satisfied by particles along the $x$-direction. Namely, $v$ and $x$ are the component of spatial velocity vector and position vector respectively, and $X$ is constant, and

$$
C=\int_{-\infty}^{+\infty} f d v^{\prime},\langle v\rangle=\frac{1}{C} \int_{-\infty}^{+\infty} f v^{\prime} d v^{\prime}, C T=\int_{-\infty}^{+\infty} f v^{\prime 2} d v^{\prime}
$$

From $v^{\prime}=v-\langle v\rangle$, we can obtain $d v^{\prime}=d v$. Then we use $t, x$ and $v^{\prime}$ to express Eq. (2) and obtain:

$$
\frac{\partial f}{\partial t}+\left(v^{\prime}+\langle v\rangle\right) \frac{\partial f}{\partial x}-\left\{\frac{\partial\langle v\rangle}{\partial t}+\left(v^{\prime}+\langle v\rangle\right) \frac{\partial\langle v\rangle}{\partial x}-X\right\} \frac{\partial f}{\partial v^{\prime}}=-\hat{v}\left(f-\hat{f}^{(0)}\right)
$$

According to HPM, we determine the nonlinear operator for Eq. (3), which is 


$$
N\left[f\left(x, v^{\prime}, t\right)\right]=\frac{\partial f}{\partial t}-\left\{\frac{\partial\langle v\rangle}{\partial t}+\left(v^{\prime}+\langle v\rangle\right) \frac{\partial\langle v\rangle}{\partial x}-X\right\} \frac{\partial f}{\partial v^{\prime}}+\hat{v}\left(f-\hat{f}^{(0)}\right)+\left(v^{\prime}+\langle v\rangle\right) \frac{\partial f}{\partial x}
$$

Assume that the assistant linear operator for Eq. (3) is $L[f]$, in order to ensure the dimensional accordance of the homotopy transformational equation and let the solution of $L[f]=0$ contain the basic structure $\exp \left(-v^{\prime 2} / 2 T\right)$, the determined assistant linear operator for Eq. (3) is

$$
L\left[f\left(x, v^{\prime}, t\right)\right]=t_{0}\left(T \frac{\partial^{2} f}{\partial v^{\prime 2}}+v^{\prime} \frac{\partial f}{\partial v^{\prime}}+f\right)
$$

where $t_{0}$ is a undetermined constant, and its dimension is $t^{-1}$.

According to HPM, we construct the following homotopy

$$
H(F, p)=(1-p)\left[L(F)-L\left(f_{0}\right)\right]-p \cdot N[F]=0 \text {. }
$$

where $p \in(0,1)$ is an embedding parameter; $F=F\left(x, v^{\prime}, t ; p\right)$ is a unknown function; $f_{0}=f_{0}\left(x, v^{\prime}, t\right)$ is an initial approximate solution of Eq. (3). The following function is chosen as the initial approximate solution of Eq. (3)

$$
f_{0}=f_{0}\left(x, v^{\prime}, t\right)=\hat{f}^{(0)}=\frac{C}{(2 \pi T)^{1 / 2}} \exp \left(-\frac{v^{\prime 2}}{2 T}\right) \text {. }
$$

By expanding Eq. (6), the following equation is obtained

$$
\begin{aligned}
& t_{0}\left(T \frac{\partial^{2} F}{\partial v^{\prime 2}}+v^{\prime} \frac{\partial F}{\partial v^{\prime}}+F\right)-t_{0}\left(T \frac{\partial^{2} f_{0}}{\partial v^{\prime 2}}+v^{\prime} \frac{\partial f_{0}}{\partial v^{\prime}}+f_{0}\right) \\
& -p t_{0}\left(T \frac{\partial^{2} F}{\partial v^{\prime 2}}+v^{\prime} \frac{\partial F}{\partial v^{\prime}}+F\right)+p t_{0}\left(T \frac{\partial^{2} f_{0}}{\partial v^{\prime 2}}+v^{\prime} \frac{\partial f_{0}}{\partial v^{\prime}}+f_{0}\right) \\
& -p\left[\left(v^{\prime}+\langle v\rangle\right) \frac{\partial f}{\partial x}-\left(\frac{\partial\langle v\rangle}{\partial t}+\left(v^{\prime}+\langle v\rangle\right) \frac{\partial\langle v\rangle}{\partial x}-X\right) \frac{\partial f}{\partial v^{\prime}}+\frac{\partial f}{\partial t}+\hat{v}\left(F-\hat{f}^{(0)}\right)\right]=0
\end{aligned}
$$

The imbedding parameter $p$ is used as a "small parameter", and the solution of Eq. (8) can be expressed as:

$$
F=F_{0}+F_{1} \cdot p+F_{2} \cdot p^{2}+\mathrm{L}
$$

By substituting Eq. (9) into Eq. (8) and comparing the coefficient of each power of $p$, the following equations are obtained

$$
p^{0}: t_{0}\left(T \frac{\partial^{2} F_{0}}{\partial v^{\prime 2}}+v^{\prime} \frac{\partial F_{0}}{\partial v^{\prime}}+F_{0}\right)-t_{0}\left(T \frac{\partial^{2} f_{0}}{\partial v^{\prime 2}}+v^{\prime} \frac{\partial f_{0}}{\partial v^{\prime}}+f_{0}\right)=0
$$




$$
\begin{aligned}
p^{1}: & t_{0}\left(T \frac{\partial^{2} F_{1}}{\partial v^{\prime 2}}+v^{\prime} \frac{\partial F_{1}}{\partial v^{\prime}}+F_{1}\right)-t_{0}\left(T \frac{\partial^{2} F_{0}}{\partial v^{\prime 2}}+v^{\prime} \frac{\partial F_{0}}{\partial v^{\prime}}+F_{0}\right)+t_{0}\left(T \frac{\partial^{2} f_{0}}{\partial v^{\prime 2}}+v^{\prime} \frac{\partial f_{0}}{\partial v^{\prime}}+f_{0}\right) \\
& -\left[\frac{\partial F_{0}}{\partial t}+\left(v^{\prime}+\langle v\rangle\right) \frac{\partial F_{0}}{\partial x}-\left(\frac{\partial\langle v\rangle}{\partial t}+\left(v^{\prime}+\langle v\rangle\right) \frac{\partial\langle v\rangle}{\partial x}-X\right) \frac{\partial F_{0}}{\partial v^{\prime}}+\hat{v}\left(F_{0}-\hat{f}^{(0)}\right)\right]=0
\end{aligned}
$$

From Eqs. (10) and (7), we know

$$
F_{0}=f_{0}=\hat{f}^{(0)}=\frac{C}{(2 \pi T)^{1 / 2}} \exp \left(-\frac{v^{\prime 2}}{2 T}\right)
$$

Then, Eq. (11) can be simplified as

$$
t_{0}\left(T \frac{\partial^{2} F_{1}}{\partial v^{\prime 2}}+v^{\prime} \frac{\partial F_{1}}{\partial v^{\prime}}+F_{1}\right)-\left[\frac{\partial f_{0}}{\partial t}+\left(v^{\prime}+\langle v\rangle\right) \frac{\partial f_{0}}{\partial x}-\left(\frac{\partial\langle v\rangle}{\partial t}+\left(v^{\prime}+\langle v\rangle\right) \frac{\partial\langle v\rangle}{\partial x}-X\right) \frac{\partial f_{0}}{\partial v^{\prime}}\right]=0
$$

By computing the mass, momentum and energy equation for Eq. (3), the following equations are got

$$
\begin{aligned}
& \frac{\partial C}{\partial t}=-C \frac{\partial\langle v\rangle}{\partial x}-\langle v\rangle \frac{\partial C}{\partial x} . \\
& \frac{\partial\langle v\rangle}{\partial t}=X-\frac{T}{C} \frac{\partial C}{\partial x}-\frac{\partial T}{\partial x}-\langle v\rangle \frac{\partial\langle v\rangle}{\partial x} . \\
& \frac{\partial T}{\partial t}=-2 T \frac{\partial\langle v\rangle}{\partial x}-\langle v\rangle \frac{\partial T}{\partial x} .
\end{aligned}
$$

and

$$
\begin{aligned}
& \frac{\partial f_{0}}{\partial t}=\frac{\partial f_{0}}{\partial C} \frac{\partial C}{\partial t}+\frac{\partial f_{0}}{\partial\langle v\rangle} \frac{\partial\langle v\rangle}{\partial t}+\frac{\partial f_{0}}{\partial T} \frac{\partial T}{\partial t} . \\
& \frac{\partial f_{0}}{\partial x}=\frac{\partial f_{0}}{\partial C} \frac{\partial C}{\partial x}+\frac{\partial f_{0}}{\partial\langle v\rangle} \frac{\partial\langle v\rangle}{\partial x}+\frac{\partial f_{0}}{\partial T} \frac{\partial T}{\partial x} .
\end{aligned}
$$

From Eqs. (14)-(18), we obtain

$$
\begin{aligned}
& {\left[\frac{\partial f_{0}}{\partial t}+\left(v^{\prime}+\langle v\rangle\right) \frac{\partial f_{0}}{\partial x}-\left(\frac{\partial\langle v\rangle}{\partial t}+\left(v^{\prime}+\langle v\rangle\right) \frac{\partial\langle v\rangle}{\partial x}-X\right) \frac{\partial f_{0}}{\partial v^{\prime}}\right]=\left(\frac{\partial \hat{f}^{(0)}}{\partial C} \frac{\partial C}{\partial t}+\frac{\partial \hat{f}^{(0)}}{\partial T} \frac{\partial T}{\partial t}\right)-\frac{\partial \hat{f}^{(0)}}{\partial v^{\prime}} \frac{\partial\langle v\rangle}{\partial t}} \\
& +\left(v^{\prime}+\langle v\rangle\right)\left[-\frac{\partial \hat{f}^{(0)}}{\partial v^{\prime}} \frac{\partial\langle v\rangle}{\partial x}+\left(\frac{\partial \hat{f}^{(0)}}{\partial C} \frac{\partial C}{\partial x}+\frac{\partial \hat{f}^{(0)}}{\partial T} \frac{\partial T}{\partial x}\right)\right]+X \frac{\partial \hat{f}^{(0)}}{\partial v^{\prime}}=\left(\frac{v^{\prime 2}}{2 T}-\frac{3}{2}\right) \frac{v^{\prime}}{T} \frac{\partial T}{\partial x} \hat{f}^{(0)}
\end{aligned} .
$$

By substitution of Eq. (19) into Eq. (13), the following equation is got

$$
t_{0}\left(T \frac{\partial^{2} F_{1}}{\partial v^{\prime 2}}+v^{\prime} \frac{\partial F_{1}}{\partial v^{\prime}}+F_{1}\right)-\left(\frac{v^{\prime 2}}{2 T}-\frac{3}{2}\right) \frac{v^{\prime}}{T} \frac{\partial T}{\partial x} \hat{f}^{(0)}=0
$$

By solving Eq. (20), we obtain 


$$
F_{1}\left(x, v^{\prime}, t\right)=\frac{v^{\prime}}{2 T t_{0}}\left(1-\frac{v^{\prime 2}}{3 T}\right) \frac{\partial T}{\partial x} \hat{f}^{(0)}
$$

Therefore, the first-order approximate solution of Eq. (6) is

$$
F=F_{0}+F_{1} \cdot p=\hat{f}^{(0)}+p \cdot \frac{v^{\prime}}{2 T t_{0}}\left(1-\frac{v^{\prime 2}}{3 T}\right) \frac{\partial T}{\partial x} \hat{f}^{(0)} .
$$

According to the basic idea of HPM and Eq. (22), we obtain

$$
f=f\left(x, v^{\prime}, t\right)=\lim _{p \rightarrow 1} F=\left[1+\frac{v^{\prime}}{2 T t_{0}}\left(1-\frac{v^{\prime 2}}{3 T}\right) \frac{\partial T}{\partial x}\right] \hat{f}^{(0)} .
$$

Formula (23) is the HPM solution of Boltzman equation with one-dimensional BGK model.

\section{Discussion}

By applying homotopy perturbation method (HPM), we obtained the first-order approximate solution (expression (23)) of Blotzmann equation with one -dimensional BGK model (Eq. (3)). By substitution of these solutions into the constraint conditions, the accuracy, reliability and rationality of the proposed solutions are verified.

For the first-order approximate solution of Eq. (3), namely expression (23), from the definition of $C,\langle v\rangle$ and $T$, and the expression of $\hat{f}^{(0)}$ we can obtain:

$$
C=\int_{-\infty}^{+\infty} f d v^{\prime}=\int_{-\infty}^{+\infty} f_{0} d v^{\prime}, C \bar{v}=\int_{-\infty}^{+\infty} f v^{\prime} d v^{\prime}=\int_{-\infty}^{+\infty} f_{0} v^{\prime} d v^{\prime}, C T=\int_{-\infty}^{+\infty} f v^{\prime 2} d v^{\prime}=\int_{-\infty}^{+\infty} f_{0} v^{\prime 2} d v^{\prime}
$$

Therefore, they require:

$$
\int_{-\infty}^{+\infty} F_{1} d v^{\prime}=0, \int_{-\infty}^{+\infty} F_{1} v^{\prime} d v^{\prime}=0, \int_{-\infty}^{+\infty} F_{1} v^{2} d v^{\prime}=0
$$

It is easy to verify that formula (21) satisfies all the constraint conditions in expression (24), hence the first-order approximate solution of Eq. (3), namely expression (23) satisfies the quality, momentum and energy conservation equations.

\section{Conclusions}

According to the kinetics theory, we applied the Boltzmann equation to describe the motion of particles in solid-gas two-phase flow. Firstly, we considered the simple situation of the Boltzmann equation with one-dimensional BGK model and applied homotopy perturbation method (HPM) to solve it. Through selecting Maxwell velocity distribution function as the initial approximate solution, and determining the adequate assistant linear operator, we obtained the first-order approximate analytic solution of the Boltzmann equation with one-dimensional BGK model. Through analyzing, it is proved that the obtained analytical solutions satisfy all the constraint conditions, and the structure of the obtained solutions is in accord with the structure of the solution that is obtained by traditional Chapman-Enskog method. This preliminarily shows the effectiveness and rapidness of HPM to solve the Boltzmann equation, and provide an effective method for solving other nonlinear fluid mechanic problems. The results obtained herein provide some theoretical basis for the further study of dynamic model of solid-gas two-phase flows, such as the sturzstrom of high-speed distant landslide caused by microseism and the sand storm caused by strong breeze. 


\section{Acknowledgements}

This work is supported by the China Postdoctoral Science Foundation (2013M540701), the State Key Laboratory of Geohazard Prevention and Geoenvironment Protection Foundation (SKLGP2012Z013) and the project for young and middle-aged key teachers of Chengdu University of Technology (KYGG201303).

\section{References}

[1] G.-Q.Wang, J.-R. Ni, "Kinetic theory for dilute solid/liquid two-phase flow" International Journal of Multiphase Flow, 17, 2, pp. 273-281(1991)

[2] N. Camara, H-L Lu, Y.-H. Zhao, W.-T. Liu, and X. Li, "Second order moments model and numerical simulation of dense gas-solid two-phase flow" Journal of Harbin Institute of Technology, 38, 11, pp. 2015-2018(2006).

[3] G.-s. Liu, H. Xu, H.-W. Wu, J.-D. Lu, C.-G. Zheng, "Modeling of gas-solid two-phase flow using kinetic theory" Journal of Combustion Science and Technology, 3, 2, pp. 143-149(1997)

[4] R. Hilfer, H. Besserer, "Macroscopic two-phase flow in porous media" Physica B: Condensed Matter, 279, 1, pp. 125-129(2000).

[5] G. Ahmadi, "Generalized continuum theory for multiphase suspension flows" International Journal of Engineering Science, 23, 1, pp. 1-25(1985).

[6] Z. Q. Huang, E J. Ding, "Theory and application of Boltzmann equation" Progress in physics, 6, 3, pp. 300-351(1986).

[7] L. Zhang, G.-Q. Wang, X.-D. Fu and Q.-C. Sun, "A new solution to Boltzmann equation of dilute solid-liquid two-phase flows with homotopy analysis method" Journal of Basic Science and Engineering, 17, 6, pp. 811-818(2009).

[8] Lei Wu, Jason M. Reese and Yonghao Zhang "Solving the Boltzmann equation deterministically by the fast spectral method: application to gas microflows" J. Fluid Mech.746, pp. 53-84(2014).

[9] J.-H. He, "Homotopy perturbation method: A new nonlinear analytical technique" Applied Mathematics and Computation. 135, 1, pp. 73-79(2003).

[10]L. Cveticanin, "Homotopy-perturbation method for pure nonlinear differential equation" Chaos, Solitons and Fractals. 30, 5, pp. 1221-1230(2006).

[11]Hamdi Cherif $\mathrm{M}$ et al 'Homotopy perturbation method for solving the fractional fisher' $\mathrm{s}$ equation” Int J Anal Appl. 10,1, pp.9-16(2016).

[12] A. Yildirim, "Application of the homotopy perturbation method for the Fokker-Planck equation" International Journal for Numerical Methods in Biomedical Engineering. 26, 9, pp. 1144-1154(2010). 